\title{
Rescue extracorporeal membrane oxygenation for refractory cardiogenic shock
}

\author{
Paweł Litwiński ${ }^{1}$, Artur Dębski ${ }^{2}$, Paweł Tyczyński ${ }^{2}$, Małgorzata Jasińska ${ }^{3}$, Jerzy Lichomski ${ }^{1}$, \\ Jarosław Szymański ${ }^{1}$, Mariusz Kuśmierczyk ${ }^{1}$ \\ ${ }^{1}$ Department of Cardiosurgery and Transplantology, Institute of Cardiology, Warsaw, Poland \\ ${ }^{2}$ Department of Interventional Cardiology and Angiology, Institute of Cardiology, Warsaw, Poland \\ ${ }^{3}$ Department of Anesthesiology, Institute of Cardiology, Warsaw, Poland
}

Postep Kardiol Inter 2015; 11, 4 (42): 327-329

DOI: $10.5114 /$ pwki.2015.55605

\section{Introduction}

Veno-arterial extracorporeal membrane oxygenation (VA-ECMO) has been increasingly used for bridge-to-recovery, bridge-to-decision or bridge to ventricular assist device (VAD) implantation or heart transplantation in patients with cardiogenic shock (CS).

We report a case of iatrogenic left main coronary artery (LMCA) dissection and secondary cardiogenic shock in which mechanical cardiopulmonary support with portable ECMO was used to rescue the patient after urgent surgical revascularization. Extracorporeal membrane oxygenation has the potential to improve tissue perfusion without the adverse consequences of medical therapies, consisting primarily of inotropic agents and vasopressors, such as increased myocardial oxygen demand and ischemia, arrhythmogenicity and reduction of tissue microcirculation, creating the opportunity to reduce the high mortality rates currently associated with conventionally managed patients in CS.

\section{Case report}

A 59-year-old male patient was admitted to coronary angiography for recurrent chest pain for 1 day. Based on ECG and elevated troponin T, acute coronary syndrome without ST elevation was diagnosed. He had no significant past medical history. Using the right radial approach and a Judkins diagnostic catheter, the very short LMCA was visualized with almost independent take-off of the left anterior descending coronary artery (LAD) and left circumflex coronary artery (LCX). First selective contrast injection into the LCX did not show any significant lesions. In order to selectively engage the LAD, exces- sively deep catheter intubation was performed, which caused iatrogenic LMCA dissection, propagating to the LAD and proximal LCX. Acute LAD closure and contrast extravasation was visualized (Figure 1). Immediate, percutaneous attempts to open the LAD were undertaken. However, despite support with inflated $1.25 \mathrm{~mm}$ and $2.0 \mathrm{~mm}$ balloons, it was impossible to insert any of the different guidewires into the true lumen of the LAD or diagonal branch. Thus no contrast flow in the mid/distal LAD segments was restored. The patient developed CS, requiring continuous infusion of inotropes, intubation and mechanical ventilation. Thus, he was immediately transferred to a tertiary hospital with the department of cardiac surgery, where rescue off-pump coronary artery bypass grafting (CABG) was performed just a few hours after dissection. Entering the operating room the patient had a sinus rhythm with a heart rate of 120 beats $/ \mathrm{min}$ and blood pressure of $85 / 55 \mathrm{~mm} \mathrm{Hg}$. The LAD dissection extended to the mid segment, distal to which the vein graft was sewn. The second vein graft was sewn to the obtuse marginal artery. The postoperative course was characterized by progressive impairment of the left ventricle (LV) systolic function. The LV ejection fraction (LVEF) decreased from $25 \%$ preoperatively to only $10-15 \%$ in the early postoperative period. No repeat early postCABG coronary angiography has been performed to document graft patency. Maximal troponin T was $20650 \mathrm{ng} / \mathrm{l}$ $(\mathrm{UNL}<14)$ and the peak lactate was $84 \mathrm{mg} / \mathrm{dl}(\mathrm{UNL}<20)$ on the second day. An intra-aortic balloon pump (IAPB) was inserted percutaneously through the left femoral artery. Due to progressive haemodynamic instability despite maximal medical therapy and IABP support, an ECMO was inserted on the second postoperative day

\section{Corresponding author:}

Paweł Tyczyński MD, PhD, Department of Interventional Cardiology and Angiology, Institute of Cardiology, 42 Alpejska St, 04-628 Warsaw, Poland, phone: +48 696091079 , e-mail: medykpol@wp.pl

Received: 15.06.2015, accepted: 27.07.2015. 
through the full sternotomy, utilizing an $8 \mathrm{~mm}$ graft sewn to the ascending aorta and a VAD venous cannulae introduced into the right atrium to salvage the patient. The ECMO circuit was based on a centrifugal pump and a hollow-fiber membrane oxygenator. All circuit components were heparin surface coated. After surgical bleeding had been controlled, continuous infusion of heparin was initiated with the activated partial thromboplastin time target of 40-60 s. The pump flow was maintained at $2.5 \mathrm{l} / \mathrm{min} / \mathrm{m}^{2}$, obtaining a mean systemic pressure of 60-70 mm Hg. Packed red blood cells and fresh frozen plasma were transfused as required. Haematocrit was maintained above $30 \%$ to optimize oxygen delivery. No exploration for bleeding was necessary. The intensive care unit course was characterized by modest but continuous clinical improvement and LVEF increase up to 35$40 \%$. The decision for ECMO weaning was undertaken on stable haemodynamic and inotropic support, with echo- cardiographic absence of tamponade and of left heart distension. Weaning was done in the operating room. The ECMO flow was gradually reduced under continuous transesophageal echocardiography monitoring of LV function and finally stopped 14 days after its initiation. Simultaneously systemic blood pressure, filling pressures and cardiac output were monitored during the process. The patient was decannulated after cardiac function stabilization and adequate gas exchange was confirmed. He was extubated on the first postoperative day, and discharged after 35 days.

During 4 months of follow-up the patient required three hospitalizations for exacerbation of heart failure. The NT-pro-BNP varied from $4883 \mathrm{pg} / \mathrm{ml}$ to $15000 \mathrm{pg} /$ $\mathrm{ml}$. Peak oxygen uptake was only $8.3 \mathrm{ml} / \mathrm{min} / \mathrm{kg}$ (34\% of predicted normal value) on the cardiopulmonary exercise testing. Thus, the patient is considered a candidate for orthotopic heart transplantation (OHT).
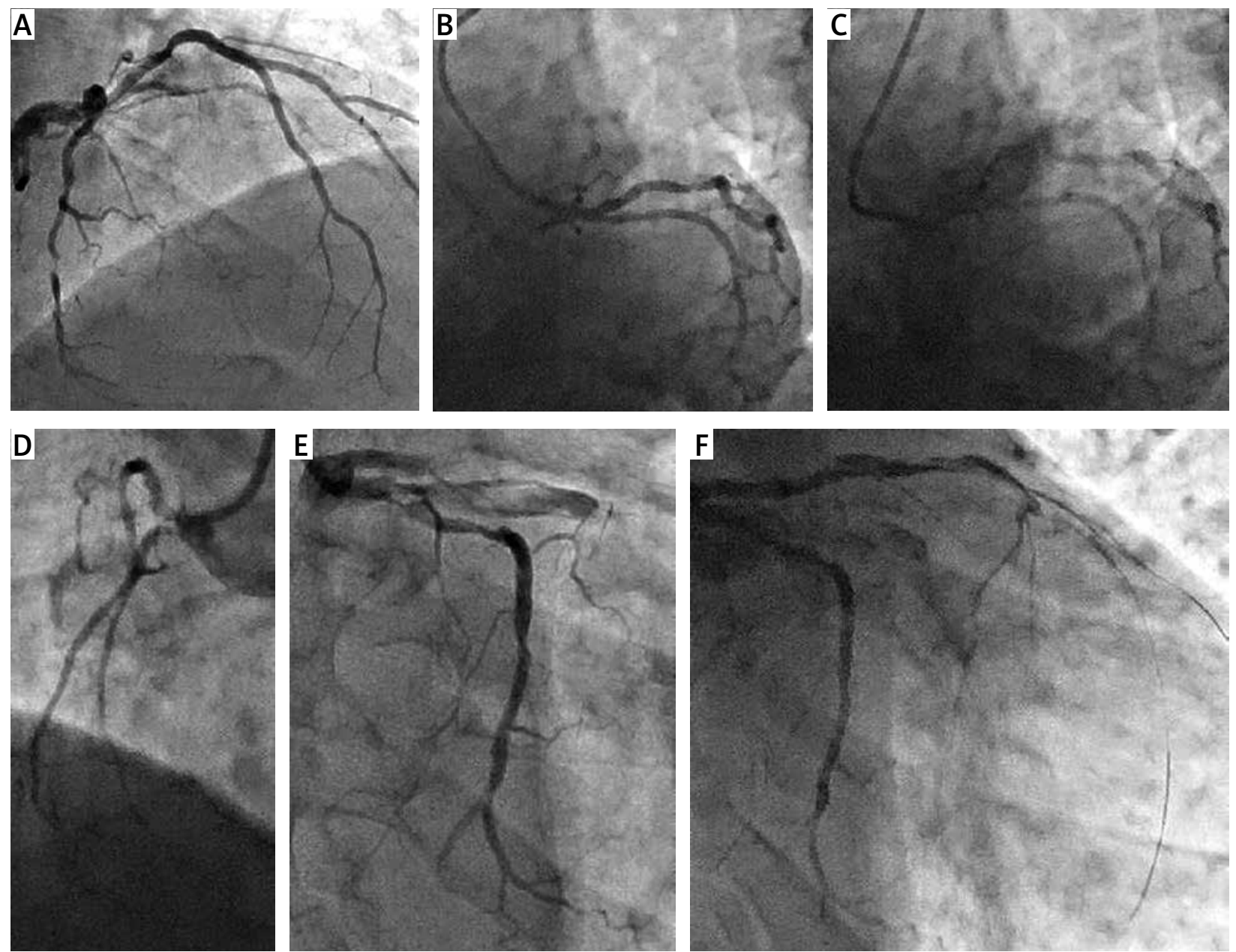

Figure 1. Angiography. A - LCA with tight lesion in LCX; B and C - Same, left caudal projections of LCA. Practically independent take-offs of LAD and LCX. Very deep catheter intubation in image "C". White arrows indicate the catheter tip, black arrows indicate the dissection. D - Small right coronary artery, $\mathbf{E}$ - latrogenic dissection of the left main coronary artery with acute LAD closure and contrast extravasation, F - Unsuccessful, percutaneous attempt to restore flow in $L A D$

$L A D$ - Left anterior coronary artery, LCA - left coronary artery, LCX - left circumflex coronary artery. 


\section{Discussion}

latrogenic LMCA dissection constitutes a rare complication ( $<1 \%$ ), although with potentially disastrous consequences. Immediate coronary blood flow restoration is the key factor for successful therapy, although percutaneous attempts to open the arteries may fail. Benefits of IABP for CS secondary to myocardial infarction are controversial, unless mechanical complications occur. After the negative SHOCK-II trial only short-term IABP use may be considered. The IABP is not effective in patients with extremely reduced LV systolic function, arrhythmias and in patients with severe and critical coronary stenoses [1]. VA-ECMO is indicated in patients with refractory CS who have an underlying potentially reversible LV dysfunction, although it can also be used as a bridge to VAD or OHT. The advantage of ECMO over percutaneous VAD is related to the possibility of bi-ventricular support and the potential to support patients with concomitant lung injury if needed. There are no randomized controlled trials comparing ECMO with other mechanical circulatory support systems in CS complicating acute myocardial infarction. However, several nonrandomized studies suggest a survival advantage from the early use of ECMO in such cases [2, 3]. Observational studies and review publications report that at least $40-50 \%$ of these patients survived to discharge after ECMO support for acute CS for various reasons [4-6]. It is crucial to underscore that such patients would probably have died without any mechanical support. In contrast to chronic heart failure, VA-ECMO for acute setting of CS may offer a considerable chance of recovery [7]. The optimal time of ECMO initiation remains unproven. However, both a longer period between cardio-pulmonary resuscitation initiation and ECMO commencement and a high pre-ECMO serum lactate level were reported to be risk factors for poor outcome [8]. Duration of ECMO therapy varies between studies. In one of the largest observational studies, mean ECMO support was 4.7 days [9], which was far below the ECMO duration in our patient. Furthermore, longer ECMO support time is one of the risk factors of mortality [10], which may just reflect the poorer haemodynamic condition of the patient. On the other hand, ECMO does have an inherent myriad of possible complications, which can be machine-related or patient-related. Haemostatic complications dominate, with bleeding and thrombosis being the most common problems. In a meta-analysis of 20 studies between 2000 and 2012, incorporating 1866 patients with ECMO for CS or cardiac arrest, complication rates were reported as follows: lower extremity ischemia (in cases with femoral cannulation), $16.9 \%$; fasciotomy or compartment syndrome, $10.3 \%$; lower extremity amputation, 4.7\%; stroke, 5.9\%; major or significant bleeding, $40.8 \%$; re-thoracotomy for post-cardiotomy bleeding or tamponade, 41.9\%; significant infection, 30.4\% [11]; and left ventricular distension despite sufficient extracorpo- real circulatory support that can jeopardize ventricular recovery and lead to pulmonary edema and ventricular thrombus formation (need for left ventricular venting).

\section{Conclusions}

VA-ECMO may be an effective treatment for selected patients in acute, refractory CS. Heart team cooperation and expertise are the prerequisites.

\section{Conflict of interest}

The authors declare no conflict of interest.

\section{References}

1. Westaby S, Anastasiadis K, Wieselthaler GM. Cardiogenic shoch in ACS. Part 2: role of mechanical circulatory support. Nat Rev Cardiol 2012; 9: 195-208.

2. Sakamoto S, Taniguchi N, Nakajima S, et al. Extracorporeal life support for cardiogenic shock or cardiac arrest due to acute coronary syndrome. Ann Thorac Surg 2012; 94: 1-7.

3. Sheu J, Tsai TH, Lee FY, et al. Early extracorporeal membrane oxygenator-assisted primary percutaneous coronary intervention improved 30-day clinical outcomes in patients with ST-segment elevation myocardial infarction complicated with profound cardiogenic shock. Crit Care Med 2010; 38: 1810-7

4. Combes A, Leprince P, Luyt CE, et al. Outcomes and long-term quality-of-life of patients supported by extracorporeal membrane oxygenation for refractory cardiogenic shock. Crit Care Med 2008; 36: 1404-11.

5. Esper SA, Bermudez C, Dueweke EJ, et al. Extracorporeal membrane oxygenation support in acute coronary syndromes complicated by cardiogenic shock. Catheter Cardiovasc Interv 2015; 86 Suppl 1: S45-50.

6. Pyka L, Pres D, Przybylski R, et al. Mechanical circulatory support in cardiogenic shock - what every interventional cardiologist should know. Postep Kardiol Inter 2014; 10: 195-200.

7. Tarzia V, Bortolussi G, Bianco R, et al. Extracorporeal life support in cardiogenic shock: impact of acute versus chronic etiology on outcome. J Thorac Cardiovasc Surg 2015; 150: 333-40.

8. Kim H, Lim SH, Hong J, et al. Efficacy of veno-arterial extracorporeal membrane oxygenation in acute myocardial infarction with cardiogenic shock. Resuscitation 2012; 83: 971-5.

9. Lee $\mathrm{SH}$, Chung $\mathrm{CH}$, Lee JW, et al. Factors predicting early- and long-term survival in patients undergoing extracorporeal membrane oxygenation (ECMO). J Card Surg 2012; 27: 255-63.

10. Chung ES, Lim C, Lee HY, et al. Results of Extracorporeal Membrane Oxygenation (ECMO) Support before coronary reperfusion in cardiogenic shock with acute myocardial infarction. Korean J Thorac Cardiovasc Surg 2011; 44: 273-8.

11. Cheng R, Hachamovitch R, Kittleson M, et al. Complications of extracorporeal membrane oxygenation for treatment of cardiogenic shock and cardiac arrest: a meta-analysis of 1866 adult patients. Ann Thorac Surg 2014; 97: 610-6. 